\title{
INVOLUTORIAL TRANSFORMATIONS IN $S_{3}$ OF ORDER $n$ WITH AN (n-1)-FOLD LINE*
}

BY

\author{
LEAMAN A. DYE
}

1. Introduction. Montesano $\dagger$ has given a brief synthetic discussion of the existence of involutorial transformations $I_{n}$ of order $n$ with an $(n-1)$-fold line $l$. He showed that the planes through $l$ are interchanged in pairs by $I_{n}$ and that the lines in one plane are transformed into lines in the conjugate plane. He also showed that the $I_{n}$ could be defined by the aid of two curves of order $n-1$ situated on the fundamental surface $F_{n-1}: l^{n-2}$ which is the image of $l$.

In this paper the $F_{n-1}: l^{n-2}$ and an $F_{n}: l^{n-1}$ are used to define an involutorial transformation of order $2 n-1$ with a $(2 n-3)$-fold line which, if certain conditions are satisfied, reduces to an involutorial transformation of order $n$ with an $(n-1)$-fold line. The explicit analytical forms of $I_{3}$ and $I_{4}$ are found by this method. For larger values of $n$ it is convenient to define $I_{n}$ by other means. There is a net of surfaces of order $m, m+1$, or $m+2$ according as $n=3 m-1,3 m$, or $3 m+1$, which is transformed by $I_{n}$ into a net of surfaces of order $m$. These nets are used to define the involutorial transformation and the equations of $I_{5}, I_{6}$, and $I_{7}$ are derived. A method is given for mapping $I_{n}$ on ordinary space so that it is apparent that $I_{n}$ is rational.

2. The birational transformation of type $(n, n)$ with an $(n-1)$-fold line. Two surfaces $F_{n}$ of order $n$ having an $(n-1)$-fold line $l$ in common, meet in a residual curve $C_{2 n-1}$. Any plane through $l$ meets each $F_{n}$ in a residual line, and the two lines meet in a point on the $C_{2 n-1}$. Hence the $C_{2 n-1}$ meets $l$ in $2 n-2$ points. If the two surfaces have a $C_{n}$ in common meeting $l$ in $n-1$ points, then by Noether's $\ddagger$ formulas the $C_{2 n-1}$ will consist of the $C_{n}$ and $n-1$ lines $l_{i}$ meeting $l . \S$ Conversely through $n-1$ lines $l_{i}$ meeting $l$, pass $\infty^{n+2}$ surfaces $F^{n}: l^{n-1}, \Sigma l_{i}$ such that any two meet in a $C_{n}$ which meets the line $l$ in $n-1$ points and each line $l_{i}$ in one point. Three of these surfaces meet in $n$ points and if we fix $n-1$ of the points, we have a homoloidal web of surfaces $F_{n}: l^{n-1}, \Sigma l_{i}, \Sigma P_{i}$.

* Presented to the Society, February 22, 1930; received by the editors January 20, 1930.

$\dagger$ D. Montesano, Su una classe di trasformazioni involutorie dello spazio, Istituto Lombardo, Rendiconti, (2), vol. 21 (1888), pp. 688-690.

$\ddagger$ M. Noether, Sulle curve multiple di superficie algebriche, Annali di Matematica, (2), vol. 5 (1871), pp. 163-177.

$\S$ H. P. Hudson, Cremona Transformations in Plane and Space, p. 316. 
There exists therefore a Cremona transformation $T_{n, n}$ between two spaces $(x)$ and $\left(x^{\prime}\right)$ by which a plane in $\left(x^{\prime}\right) \sim$ a surface of the web of $F_{n}$ in $(x)$. A plane and a surface $F_{n}$ in the $(x)$ space $\sim$ a surface $F_{n}^{\prime}$ and a plane in the $\left(x^{\prime}\right)$ space. The curves of intersection correspond and have the same genus.* Hence a plane section of $F_{n}^{\prime}$ is a $C_{n}^{\prime}$ with an $(n-1)$-fold point and the surfaces $F_{n}^{\prime}$ must have a common $(n-1)$-fold line $l^{\prime}$. Two planes in $(x) \sim$ two $F_{n}^{\prime}$ in $\left(x^{\prime}\right)$ meeting in a residual $C_{2 n-1}^{\prime}$ which meets $l^{\prime}$ in $2 n-2$ points. The line of intersection of the two planes $\sim$ a non-composite $C_{n}^{\prime}$ which can meet $l^{\prime}$ in not more than $n-1$ points. Therefore the residual $C_{n-1}^{\prime}$ must meet $l^{\prime}$ in $n-1$ points, i.e. $C_{n-1}^{\prime}$ consists of $n-1$ lines $l_{i}^{\prime}$. The $C_{n}^{\prime}$ meets $l^{\prime}$ in exactly $n-1$ points and each $l_{i}^{\prime}$ in one point. Three such surfaces $F_{n}^{\prime}$ meet in $n$ points of which $n-1$ must be fixed in order to have a homoloidal web.

Among the surfaces of the web in the $(x)$ space there is a pencil consisting of a plane through $l$ and the fixed $F_{n-1}: l^{n-2}, \Sigma l_{i}, \Sigma P_{i}$. Therefore there is a pencil of planes through $l^{\prime}$ in $\left(x^{\prime}\right)$ which corresponds to this pencil of the web of $F_{n}$, and $l^{\prime} \sim$ the fixed $F_{n-1}$.

A general line in $\left(x^{\prime}\right) \sim$ a $C_{n}: \Sigma P_{i}$, hence $P_{i} \sim$ a plane. Since $C_{n}$ meets each $l_{i}$ once, $l_{i} \sim$ a plane. The plane $\sigma_{i}$ through $l$ and $P_{i} \sim$ a plane $\rho_{i}^{\prime}$ through $l^{\prime}$, but since $P_{i} \sim$ a plane, $P_{i} \sim \rho_{i}^{\prime}$, and the plane $\sigma_{i}$ apart from $P_{i}$ and $l \sim$ a curve $s^{\prime}$ in $\rho_{i}^{\prime}$. A general plane of $\left(x^{\prime}\right)$ meets $\rho_{i}^{\prime}$ in a line $L^{\prime}$ and the curve $s^{\prime}$ in one or more points $Q^{\prime}$. The corresponding $F_{n}$ meets $\sigma_{i}$ in one line $L$ through $P_{i}$. Therefore the curve $s^{\prime}$ is a line. The line $L^{\prime}$, except for the point $Q^{\prime}, \sim P_{i}$ and $Q^{\prime} \sim L$. The line $s^{\prime}$ must be a fundamental line $l_{i}^{\prime}$ because the points of $s^{\prime} \sim$ lines in $\sigma_{i}$ through $P_{i}$. In a similar manner it is seen that $l_{i} \sim$ a plane $\sigma_{i}^{\prime}$ through $l^{\prime}$ and $P_{i}^{\prime}$. The plane $\rho_{i}$ through $l$ and $l_{i} \sim$ the point $P_{i}^{\prime}$ in $\sigma_{i}^{\prime}$. Therefore the points and lines $P_{i}$ and $l_{i}$ are associated in pairs with the lines and points $l_{i}^{\prime}$ and $P_{i}^{\prime}$ respectively.

3. The involutorial transformation. When the two spaces are superimposed for the involutorial case, the fundamental systems must coincide and the planes through $l$ are interchanged in pairs by the involutorial transformation $I_{n}$. If $x_{1}=0$ and $x_{2}=0$ are the invariant planes of this pencil, the four equations of $I_{n}$ can be obtained from three homogeneous equations of the following form:

$$
\begin{aligned}
& x_{1}^{\prime}=x_{1} \\
& x_{2}^{\prime}=-x_{2} \\
& x_{3}^{\prime}=\left(d+e x_{3}+f x_{4}\right) /\left(a+b x_{3}+c x_{4}\right),
\end{aligned}
$$

* G. Loria, Sulla classificazione delle trasformazioni di genere zero, Istituto Lombardo, Rendiconti, (2), vol. 23 (1890), pp. 824-834. 
where $a=a\left(x_{1}, x_{2}\right)$, etc., $\left(d+e x_{3}+f x_{4}\right)=0$ is any $F_{n}$ of the web, and $\left(a+b x_{3}\right.$ $\left.+c_{4}\right)=0$ is the fixed $F_{n-1}$. Since we are dealing with involutorial transformations the inverse of equations (1) have the same form as (1). If in the inverse of $\left(1_{3}\right)$ we replace $x_{1}^{\prime}, x_{2}^{\prime}$ by $x_{1},-x_{2}$ we have

$$
x_{3}=\left(\bar{d}+\bar{e} x_{3}^{\prime}+\bar{f} x_{4}^{\prime}\right) /\left(\bar{a}+\bar{b} x_{3}^{\prime}+\bar{c} x_{4}^{\prime}\right),
$$

where $\bar{a}=a\left(x_{1},-x_{2}\right)$, etc. This equation can be solved for $x_{4}^{\prime}$ and thus we get the fourth equation of the involutorial transformation as

$$
\begin{aligned}
x_{4}^{\prime}= & \left\{\left[(\bar{e} d+\bar{d} a)-x_{3}(\bar{b} d+\bar{a} a)\right]+x_{3}\left[(\bar{e} e+\bar{d} b)-x_{3}(\bar{b} e+\bar{a} b)\right]\right. \\
& \left.+x_{4}\left[(\bar{e} f+\bar{d} c)-x_{3}(\bar{b} f+\bar{a} c)\right]\right\} /\left[\left(a+b x_{3}+c x_{4}\right)\left(\bar{c} x_{3}-\bar{f}\right)\right] .
\end{aligned}
$$

When the conditions that $\bar{c} x_{3}-\bar{f}$ be a factor of the numerator are satisfied and this factor is removed, we have the $I_{n}$ with an $(n-1)$-fold line, defined analytically.

4. The cubic case. A non-homogeneous coördinate system is useful in the cases when $n=3$ or 4 , so we put $x_{2} / x_{1}=\lambda, x_{3} / x_{1}=x$, and $x_{4} / x_{1}=y$. When $n=3$ there are only two fundamental points $P_{1}, P_{2}$; any plane through the line joining them is transformed by $I_{3}$ into another such plane and the two planes $\rho_{i}=0$, which are the planes $l, l_{i}$. Among the planes of the pencil on the line $P_{1} P_{2}$ there are at least two which are invariant. Let $x=0$ and $y=0$ be two of the invariant planes, and let $\rho_{i} \equiv \lambda_{i}-\lambda=0$. The points $P_{1}, P_{2}$ are then determined by the planes $\sigma_{i} \equiv \lambda_{i}+\lambda=0$ and the line $x=y=0$. One surface of the web is $\rho_{1} \rho_{2} x=0$, and the equation of the fixed quadric determined by $l, 2 l_{i}, 2 P_{i}$ is of the form

$$
\sigma_{1} \sigma_{2}+\left(a_{0}+a_{1} \lambda\right) x+\left(b_{0}+b_{1} \lambda\right) y=0 .
$$

We can write the first two equations of $I_{3}$ as follows:

$$
\begin{aligned}
& \lambda^{\prime}=-\lambda, \\
& x^{\prime}=h \rho_{1} \rho_{2} x /\left\{\sigma_{1} \sigma_{2}+\left(a_{0}+a_{1} \lambda\right) x+\left(b_{0}+b_{1} \lambda\right) y\right\} .
\end{aligned}
$$

If we write the inverse of $\left(4_{2}\right)$ and replace $\lambda^{\prime}$ by $-\lambda$ we have

$$
x=h \sigma_{1} \sigma_{2} x^{\prime} /\left\{\rho_{1} \rho_{2}+\left(a_{0}-a_{1} \lambda\right) x^{\prime}+\left(b_{0}-b_{1} \lambda\right) y^{\prime}\right\} .
$$

When (5) is solved for $y^{\prime}$, it has the form

$$
\begin{aligned}
y^{\prime}=\rho_{1} \rho_{2} & \left\{\left(h^{2}-1\right) \sigma_{1} \sigma_{2}-x\left[a_{0}(h+2)-a_{1}(h-1) \lambda\right]\right. \\
& \left.-y\left(b_{0}+b_{1} \lambda\right)\right\} /\left[\left\{\sigma_{1} \sigma_{2}+\left(a_{0}+a_{1} \lambda\right) x+\left(b_{0}+b_{1} \lambda\right) y\right\}\left(b_{0}-b_{1} \lambda\right)\right] .
\end{aligned}
$$

Since $y=0$ is an invariant plane the first two terms in the numerator of (6) must vanish, and the coefficient of $y$ must be divisible by $b-b_{1} \lambda$. This requires that 


$$
b_{1}=0, \quad h= \pm 1, \quad a_{0}(h+1)-a_{1}(h-1) \lambda=0 .
$$

The third condition presents two cases, namely

or

$$
h=1, \quad \text { so that } a_{0}=0,
$$

$$
h=-1 \text {, so that } a_{1}=0 .
$$

The cubic involutorial transformation may now be written in the form

$$
\begin{aligned}
& \lambda^{\prime}=-\lambda, \\
& x^{\prime}=x \rho_{1} \rho_{2} /\left(\sigma_{1} \sigma_{2}+a_{1} \lambda x+b_{0} y\right), \\
& y^{\prime}=-y \rho_{1} \rho_{2} /\left(\sigma_{1} \sigma_{2}+a_{1} \lambda x+b_{0} y\right),
\end{aligned}
$$

or

$$
\begin{aligned}
& \lambda^{\prime}=-\lambda, \\
& x^{\prime}=-x \rho_{1} \rho_{2} /\left(\sigma_{1} \sigma_{2}+a_{0} x+b_{0} y\right), \\
& y^{\prime}=-y \rho_{1} \rho_{2} /\left(\sigma_{1} \sigma_{2}+a_{0} x+b_{0} y\right) .
\end{aligned}
$$

In the first case when $h=1$, the pencil of planes through $P_{1}, P_{2}$ is invariant. In the second case when $h=-1$, each plane of the pencil is invariant.

5. The quartic case. There are three fundamental points; one of the surfaces of the web consists of the plane $P_{1} P_{2} P_{3}$ and of the three planes $l, l_{i}$. We can take the points $P_{1}, P_{2}$ as in $I_{3}$ and the plane $P_{1} P_{2} P_{3}$ as $x=0$. The lines $l_{i}$ in the planes $\rho_{i} \equiv \lambda_{i}-\lambda=0$ and the points $P_{i}$ lie in the planes $\sigma_{i} \equiv \lambda_{i}$ $+\lambda=0$. The equation of the fixed $F_{3}: l^{2}, 3 l_{i}, 3 P_{i}$ may be written in the form

$$
\sigma_{1} \sigma_{2}(1-\lambda)+\left(a_{0}+a_{1} \lambda+a_{2} \lambda^{2}\right) x+\left(b_{0}+b_{1} \lambda+b_{2} \lambda^{2}\right) y=0 .
$$

The first two equations of the $I_{4}$ are now given by

(91) $\lambda^{\prime}=-\lambda$,

$\left(9_{2}\right) x^{\prime}=h \rho_{1} \rho_{2} \rho_{3} x /\left[\sigma_{1} \sigma_{2}(1-\lambda)+\left(a_{0}+a_{1} \lambda+a_{2} \lambda^{2}\right) x+\left(b_{0}+b_{1} \lambda+b_{2} \lambda^{2}\right) y\right]$.

The inverse of $\left(9_{2}\right)$ with $\lambda^{\prime}$ replaced by $-\lambda$ is

(10) $x=h \sigma_{1} \sigma_{2} \sigma_{3} x^{\prime} /\left[\rho_{1} \rho_{2}(1+\lambda)+\left(a_{0}-a_{1} \lambda^{\prime}+a_{2} \lambda^{2}\right) x^{\prime}+\left(b_{0}-b_{1} \lambda+b_{2} \lambda^{2}\right) y^{\prime}\right]$.

If (10) is solved for $y^{\prime}$ we have

$$
\begin{aligned}
y^{\prime}= & \rho_{1} \rho_{2}\left\{\sigma_{1} \sigma_{2}\left(h^{2} \rho_{3} \sigma_{3}-1+\lambda^{2}\right)-x\left[h \rho_{3}\left(a_{0}-a_{1} \lambda+a_{2} \lambda^{2}\right)\right.\right. \\
& \left.\left.+(1+\lambda)\left(a_{0}+a_{1} \lambda+a_{2} \lambda^{2}\right)\right]-y(1+\lambda)\left(b_{0}+b_{1} \lambda+b_{2} \lambda^{2}\right)\right\} /\left\{\left[b_{0}\right.\right. \\
& \left.\left.-b_{1} \lambda+b_{2} \lambda^{2}\right]\left[\sigma_{1} \sigma_{2}(1-\lambda)+\left(a_{0}+a_{1} \lambda+a_{2} \lambda^{2}\right) x+\left(b_{0}+b_{1} \lambda+b_{2} \lambda^{2}\right) y\right]\right\} .
\end{aligned}
$$

The expressions 


$$
\begin{gathered}
\sigma_{1} \sigma_{2}\left(h^{2} \rho_{3} \sigma_{3}-1+\lambda^{2}\right), \\
h \rho_{3}\left(a_{0}-a_{1} \lambda+a_{2} \lambda^{2}\right)+(1+\lambda)\left(a_{0}+a_{1} \lambda_{4} ;+a_{2} \lambda^{2}\right), \\
(1+\lambda)\left(b_{0}+b_{1} \lambda+b_{2} \lambda^{2}\right)
\end{gathered}
$$

must therefore contain the factor $b_{0}-b_{1} \lambda+b_{2} \lambda^{2}$. From (14) we find that $b_{1}=0$ and if we use this in (12) we have the condition

$$
\left(1-h^{2}\right) /\left(\lambda_{3}{ }^{2} h^{2}-1\right)=b_{2} / b_{0} .
$$

From (13) we get the conditions

$$
\begin{gathered}
{\left[a_{2}\left(1+h \lambda_{3}\right)+a_{1}(1+h)\right] /\left[a_{0}\left(1+h \lambda_{3}\right)\right]=b_{2} / b_{0},} \\
(1-h) a_{2} /\left[a_{1}\left(1-h \lambda_{3}\right)+a_{0}(1-h)\right]=b_{2} / b_{0} .
\end{gathered}
$$

These last two conditions may be rewritten as

$$
\begin{aligned}
\left(h \lambda_{3}+1\right)\left(a_{0} b_{2}-a_{2} b_{0}\right) & =a_{1} b_{0}(1+h), \\
(1-h)\left(a_{0} b_{2}-a_{2} b_{0}\right) & =a_{1} b_{2}\left(h \lambda_{3}-1\right) .
\end{aligned}
$$

If we divide $\left(17^{\prime}\right)$ by $\left(16^{\prime}\right)$ we get condition (15) over again so that (15) is included in (16) and (17). We can solve (16) and (17) for $h$ and obtain

$$
\begin{aligned}
& h=\left(a_{0} b_{2}-a_{2} b_{0}+a_{1} b_{2}\right) /\left(a_{0} b_{2}-a_{2} b_{0}+\lambda_{3} a_{1} b_{2}\right), \\
& h=\left(a_{1} b_{1}-a_{0} b_{2}+a_{2} b_{0}\right) /\left(\lambda_{3} a_{0} b_{2}-\lambda_{3} a_{2} b_{0}-a_{1} b_{0}\right) ;
\end{aligned}
$$

if we equate these values of $h$ we get

(20) $\left(\lambda_{3}+1\right)\left(a_{0} b_{2}-a_{2} b_{0}\right)^{2}+2 a_{1}\left(a_{0} b_{2}-a_{2} b_{0}\right)\left(b_{2} \lambda_{3}-b_{0}\right)-a_{1}^{2} b_{0} b_{2}\left(\lambda_{3}+1\right)=0$.

The quartic involutorial transformation is therefore determined by the equations

$$
\begin{aligned}
\lambda & =-\lambda, \\
x^{\prime} & =\frac{h x \rho_{1} \rho_{2} \rho_{3}}{\sigma_{1} \sigma_{2}(1-\lambda)+\left(a_{0}+a_{1} \lambda+a_{2} \lambda^{2}\right) x+\left(b_{0}+b_{2} \lambda^{2}\right) y} \\
y^{\prime} & =\frac{\rho_{1} \rho_{2}\left\{\sigma_{1} \sigma_{2}\left(1-h^{2}\right)-x\left[a_{2}\left(1+h \lambda_{3}\right)+a_{1}(1+h)+a_{2} \lambda(1-h)-b_{2} y(1+\lambda)\right\}\right.}{b_{2}\left[\sigma_{1} \sigma_{2}(1-\lambda)+\left(a_{0}+a_{1} \lambda+a_{2} \lambda^{2}\right) x+\left(b_{0}+b_{2} \lambda^{2}\right) y\right]} .
\end{aligned}
$$

In these equations $h$ is defined by (18) or (19) and the coefficients $a_{i}$ and $b_{i}$ are subject to condition (20).

6. The quintic case. There is a net of quadrics through $l$ and the $4 P_{i}$ which is invariant under the $I_{5}$. We can use the vertices of the tetrahedron of reference for the $4 P_{i}$ and take 


$$
\begin{aligned}
& X_{1} \equiv d_{1} x_{1}+d_{2} x_{2}+d_{3} x_{3}+d_{4} x_{4}=0, \\
& X_{2} \equiv x_{1}+x_{2}+x_{3}+x_{4}=0
\end{aligned}
$$

as the invariant planes through $l$, so that

$$
\begin{aligned}
& x_{1}^{\prime}=X_{1} F_{4}, \\
& x_{2}^{\prime}=-X_{2} F_{4},
\end{aligned}
$$

where $F_{4}$ is determined by $l^{3}, 4 l_{i}, 4 P_{i}$. The planes $l, P_{i}$ are given by $\sigma_{i} \equiv X_{1}$ $-d_{i} X_{2}=0$ and the planes $l, l_{i}$ by $\rho_{i} \equiv X_{1}+d_{i} X_{2}=0$. The net of quadrics has the form

$$
k_{1} \sigma_{1} x_{1}+k_{2} \sigma_{2} x_{2}+k_{3} \sigma_{3} x_{3}=0
$$

and from (22) we have the identity

$$
\sigma_{1} x_{1}+\sigma_{2} x_{2}+\sigma_{3} x_{3}+\sigma_{4} x_{4}=0 .
$$

The quadrics of the net are interchanged in pairs involutorially by $I_{b}$, so that the involutorial transformation can be defined by

$$
\begin{aligned}
\left(a_{1} \sigma_{1}^{\prime} x_{1}^{\prime}+a_{2} \sigma_{2}^{\prime} x_{2}^{\prime}+a_{3} \sigma_{3}^{\prime} x_{3}^{\prime}\right)=\left(a_{1} \sigma_{1} x_{1}+a_{2} \sigma_{2} x_{2}+a_{3} \sigma_{3} x_{3}\right) \rho_{2} \rho_{3} \rho_{4} F_{4}, \\
\left(b_{1} \sigma_{1}^{\prime} x_{1}^{\prime}+b_{2} \sigma_{2}^{\prime} x_{2}^{\prime}+b_{3} \sigma_{3}^{\prime} x_{3}^{\prime}\right)=\left(b_{1} \sigma_{1} x_{1}+b_{2} \sigma_{2} x_{2}+b_{3} \sigma_{3} x_{3}\right) \rho_{1} \rho_{3} \rho_{4} F_{4}, \\
\left(c_{1} \sigma_{1}^{\prime} x_{1}^{\prime}+c_{2} \sigma_{2}^{\prime} x_{2}^{\prime}+c_{3} \sigma_{3}^{\prime} x_{3}^{\prime}\right)=-\left(c_{1} \sigma_{1} x_{1}+c_{2} \sigma_{2} x_{2}+c_{3} \sigma_{3} x_{3}\right) \rho_{1} \rho_{2} \rho_{4} F_{4},
\end{aligned}
$$

and the identity

$$
\sigma_{1}^{\prime} x_{1}^{\prime}+\sigma_{2}^{\prime} x_{2}^{\prime}+\sigma_{3}^{\prime} x_{3}^{\prime}+\sigma_{4}^{\prime} x_{4}^{\prime}=0 .
$$

If we solve (23) for $x_{i}^{\prime}$ replacing $\sigma_{i}^{\prime}$ by $\rho_{i}$ and use (24) to obtain $x_{i}^{\prime}$, we have the $I_{5}$ expressed by

$$
\begin{aligned}
& x_{1}^{\prime}=\cdot\left[\sigma_{1} x_{1} \Delta-2 C_{1}\left(c_{1} \sigma_{1} x_{1}+c_{2} \sigma_{2} x_{2}+c_{3} \sigma_{3} x_{3}\right)\right] \rho_{2} \rho_{3} \rho_{4}, \\
& x_{2}^{\prime}=\left[\sigma_{2} x_{2} \Delta-2 C_{2}\left(c_{1} \sigma_{1} x_{1}+c_{2} \sigma_{2} x_{2}+c_{3} \sigma_{3} x_{3}\right)\right] \rho_{1} \rho_{3} \rho_{4}, \\
& x_{3}^{\prime}=\left[\sigma_{3} x_{3} \Delta-2 C_{3}\left(c_{1} \sigma_{1} x_{1}+c_{2} \sigma_{2} x_{2}+c_{3} \sigma_{3} x_{3}\right)\right] \rho_{1} \rho_{2} \rho_{4}, \\
& x_{4}^{\prime}=\left[\sigma_{4} x_{4} \Delta+2\left(C_{1}+C_{2}+C_{3}\right)\left(c_{1} \sigma_{1} x_{1}+c_{2} \sigma_{2} x_{2}+c_{3} \sigma_{3} x_{3}\right)\right] \rho_{1} \rho_{2} \rho_{3},
\end{aligned}
$$

where

$$
\Delta=\left|\begin{array}{lll}
a_{1} & a_{2} & a_{3} \\
b_{1} & b_{2} & b_{3} \\
c_{1} & c_{2} & c_{3}
\end{array}\right|
$$

and $C_{i}$ is the cofactor of $c_{i}$ in $\Delta$.

7. The involutorial transformations $I_{6}, I_{7}$, and $I_{n}$. There is a net of $F_{3}: l^{2}, 5 P_{i}, l_{5}$ which is transformed into a net of $F_{2}: l, 4 P_{i}(i<5)$ by $I_{6}$. Among the cubics of the net there is the pencil of $F_{2}: l, 5 P_{i}$ with the fixed component 
$\rho_{5}=0$, which is invariant under $I_{6}$. Hence using the same coordinate system as in the quintic case we can determine the $I_{6}$ by

$$
\begin{aligned}
a_{1} \sigma_{1}^{\prime} x_{1}^{\prime}+a_{2} \sigma_{2}^{\prime} x_{2}^{\prime}+a_{3} \sigma_{3}^{\prime} x_{3}^{\prime} & =\left(a_{1} \sigma_{1} x_{1}+a_{2} \sigma_{2} x_{2}+a_{3} \sigma_{3} x_{3}\right) \rho_{1} \rho_{2} \rho_{3} \rho_{4} \rho_{5} F_{5}, \\
b_{1} \sigma_{1}^{\prime} x_{1}^{\prime}+b_{2} \sigma_{2}^{\prime} x_{2}^{\prime}+b_{3} \sigma_{3}^{\prime} x_{3}^{\prime} & =-\left(b_{1} \sigma_{1} x_{1}+b_{2} \sigma_{2} x_{2}+b_{3} \sigma_{3} x_{3}\right) \rho_{1} \rho_{2} \rho_{3} \rho_{4} \rho_{5} F_{5}, \\
c_{1} \sigma_{1}^{\prime} x_{1}^{\prime}+c_{2} \sigma_{2}^{\prime} x_{2}^{\prime}+c_{3} \sigma_{3}^{\prime} x_{3}^{\prime} & =F_{3} \rho_{1} \rho_{2} \rho_{3} \rho_{4} F_{5}, \\
d_{1} x_{1}^{\prime}+d_{2} x_{2}^{\prime}+d_{3} x_{3}^{\prime}+d_{4} x_{4}^{\prime} & =\left(d_{1} x_{1}+d_{2} x_{2}+d_{3} x_{3}+d_{4} x_{4}\right) F_{5}, \\
x_{1}^{\prime}+x_{2}^{\prime}+x_{3}^{\prime}+x_{1}^{\prime} & =-\left(x_{1}+x_{2}+x_{3}+x_{4}\right) F_{5},
\end{aligned}
$$

where $F_{5}$ is the fixed quintic surface. The $a_{i}$ and $b_{i}$ are restricted since these quadrics must contain $P_{5}$. The cubic $F_{3}$ is of the form

$$
\begin{aligned}
F_{3} \equiv\left(g_{1} X_{1}^{2}+g_{2} X_{1} X_{2}+g_{3} X_{2}^{2}\right)\left(g_{4} x_{1}+g_{5} x_{2}\right. & \left.+g_{6} x_{3}\right) \\
& +\rho_{5}\left(g_{7} \sigma_{1} x_{1}+g_{8} \sigma_{2} x_{2}+g_{9} \sigma_{3} x_{3}\right)=0,
\end{aligned}
$$

where $g_{4} x_{1}+g_{5} x_{2}+g_{6} x_{3}=0$ is the plane through $l_{5}, P_{4}$; and $g_{7} \sigma_{1} x_{1}+g_{8} \sigma_{2} x_{2}$ $+g_{9} \sigma_{3} x_{3}=0$ is a quadric of the pencil $l, 5 P_{i}$. The $a_{i}, b_{i}, c_{i}, d_{i}, g_{i}$ must satisfy the conditions necessary in order that $F_{3}$ may be transformed by $I_{6}$ into

$$
\left(c_{1} \sigma_{1} x_{1}+c_{2} \sigma_{2} x_{2}+c_{3} \sigma_{3} x_{3}\right) \rho_{1} \rho_{2} \rho_{3} \rho_{4} \rho_{5} \sigma_{5} F_{5}^{2} .
$$

When $n=7$ there is a net of quartic surfaces $F_{4}: l^{3}, 6 P_{i}, l_{5}, l_{6}$ which correspond to a net of quadrics $F_{2}: l, 4 P_{i}(i<5)$. Among the surfaces of the net there is a pencil of $F_{3}: l^{2}, 6 P_{i}, l_{5}$ with $\rho_{6}=0$ as a fixed component, which is transformed into the pencil of $F_{2}: l, 5 P_{i}(i<6)$. Among the surfaces of the pencil there is the cubic consisting of the plane $\rho_{5}=0$ and the quadric $F_{2}: l$, $6 P_{i}$ which is invariant under $I_{7}$. The equations which determine the $I_{7}$ are therefore of the following form:

$$
\begin{aligned}
a_{1} \sigma_{1}^{\prime} x_{1}^{\prime}+a_{2} \sigma_{2}^{\prime} x_{2}^{\prime}+a_{3} \sigma_{3}^{\prime} x_{3}^{\prime} & =\left(a_{1} \sigma_{1} x_{1}+a_{2} \sigma_{2} x_{2}+a_{3} \sigma_{3} x_{3}\right) \rho_{1} \rho_{2} \rho_{3} \rho_{4} \rho_{5} \rho_{6} F_{6}, \\
b_{1} \sigma_{1}^{\prime} x_{1}^{\prime}+b_{2} \sigma_{2}^{\prime} x_{2}^{\prime}+b_{3} \sigma_{3}^{\prime} x_{3}^{\prime} & =F_{3} \rho_{1} \rho_{2} \rho_{3} \rho_{4} \rho_{5} F_{6}, \\
c_{1} \sigma_{1}^{\prime} x_{1}^{\prime}+c_{2} \sigma_{2}^{\prime} x_{2}^{\prime}+c_{3} \sigma_{3}^{\prime} x_{3}^{\prime} & =F_{4} \rho_{1} \rho_{2} \rho_{3} \rho_{4} F_{6} \\
d_{1} x_{1}^{\prime}+d_{2} x_{2}^{\prime}+d_{3} x_{3}^{\prime}+d_{4} x_{4}^{\prime} & =\left(d_{1} x_{1}+d_{2} x_{2}+d_{3} x_{3}+d_{4} x_{4}\right) F_{6}, \\
x_{1}^{\prime}+x_{2}^{\prime}+x_{3}^{\prime}+x_{4}^{\prime} & =-\left(x_{1}+x_{2}+x_{3}+x_{4}\right) F_{6} .
\end{aligned}
$$

The forms obtained for $n=5,6,7$ can be generalized as follows:

A. If $n=3 m-1$ there is a net of $F_{m}: l^{m-1}, \Sigma P_{i}$.

B. If $n=3 m$ there is a net of $F_{m+1}: l^{m}, \Sigma P_{i}, l_{3 m-1}$ containing the pencil of $F_{m}: l^{m-1}, \Sigma P_{i}$ with a fixed component the plane $l, l_{3 m-1}$.

C. If $n=3 m+1$ there is a net of $F_{m+2}: l^{m+1}, \Sigma P_{i}, l_{3 m-1}, l_{3 m}$ containing a pencil of $F_{m+1}: l^{m}, \Sigma P_{i}, l_{3 m-1}$ with the fixed component $l, l_{3 m}$. One of the surfaces of the pencil is the $F_{m}: l^{m-1}, \Sigma P_{i}$ with the fixed component $l, l_{3 m-1}$. 
In each case the net is transformed into a net of $F_{m}: l^{m-1}, \Sigma P_{i}(i<3 m-1)$ by $I_{n}$, hence $I_{n}$ can be defined by means of the nets.

8. The mapping of the involutorial transformation $I_{n}$. The expressions $2 x_{1} x_{1}^{\prime},-2 x_{2} x_{2}^{\prime}, x_{1} x_{3}^{\prime}+x_{3} x_{1}^{\prime}, x_{2} x_{3}^{\prime}+x_{3} x_{2}^{\prime}$ are invariant under $I_{n}$. Let us consider the correspondence between the $(x)$ space and a $(y)$ space where the values of $x_{i}^{\prime}$ above are those defined in $\$ 3,(1)$. The correspondence has the form

$$
\begin{aligned}
& y_{1}=2 x_{1}{ }^{2}\left(a+b x_{3}+c x_{4}\right), \\
& y_{2}=2 x_{2}{ }^{2}\left(a+b x_{3}+c x_{4}\right), \\
& y_{3}=x_{1}\left[\left(d+e x_{3}+f x_{4}\right)+x_{3}\left(a+b x_{3}+c x_{4}\right)\right], \\
& y_{4}=x_{2}\left[\left(d+e x_{3}+f x_{4}\right)-x_{3}\left(a+b x_{3}+c x_{4}\right)\right] .
\end{aligned}
$$

These equations can be solved for $x_{i}$ as follows:

$$
\begin{aligned}
x_{2} / x_{1} & = \pm\left(y_{2} / y_{1}\right)^{1 / 2}, \\
x_{3} & =-x_{1} \bar{U} /\left(x_{2} y_{1}\right), \\
x_{4} & =\left[d x_{2}^{2} y_{1}^{2}-a x_{1} x_{2} y_{1} U-e x_{1} x_{2} y_{1} \bar{U}+b x_{1}^{2} U \bar{U}\right] /\left\lfloor c x_{1} x_{2} y_{1} U-f x_{2}^{2} y_{1}^{2}\right\rfloor,
\end{aligned}
$$

where $U=x_{1} y_{4}+x_{2} y_{3}, \bar{U}=x_{1} y_{4}-x_{2} y_{3}$. Hence equations (26) define a $(1,2)$ correspondence.

If we rewrite the equations of the correspondence in terms of $x_{i}^{\prime}$ and replace $x_{1}^{\prime}, x_{2}^{\prime}$ by $x_{1},-x_{2}$, we have

$$
\begin{aligned}
& y_{1}=2 x_{1}^{2}\left(\bar{a}+\bar{b} x_{3}^{\prime}+\bar{c} x_{4}^{\prime}\right), \\
& y_{2}=2 x_{2}^{2}\left(\bar{a}+\bar{b} x_{3}^{\prime}+\bar{c} x_{4}^{\prime}\right), \\
& y_{3}=x_{1}\left[\left(\bar{d}+\bar{e} x_{3}^{\prime}+\bar{f} x_{4}^{\prime}\right)+x_{3}^{\prime}\left(\bar{a}+\bar{b} x_{3}^{\prime}+\bar{c} x_{4}^{\prime}\right)\right], \\
& y_{4}=-x_{2}\left[\left(\bar{d}+\bar{e} x_{3}^{\prime}+\bar{f} x_{4}^{\prime}\right)-x_{3}^{\prime}\left(\bar{a}+\bar{b} x_{3}^{\prime}+\bar{c} x_{4}^{\prime}\right)\right],
\end{aligned}
$$

where $\bar{a}=a\left(x_{1},-x_{2}\right)$, etc. If we equate the values of $y_{i}$ given in (26) and (28) we have

$$
a+b x_{3}+c x_{4}=\bar{a}+\bar{b} x_{3}^{\prime}+\bar{c} x_{4}^{\prime},
$$

$$
\begin{aligned}
\left(d+e x_{3}+f x_{4}\right)+x_{3}(a+ & \left.b x_{3}+c x_{4}\right) \\
& =\left(\bar{d}+\bar{e} x_{3}^{\prime}+\bar{f} x_{4}^{\prime}\right)+x_{3}^{\prime}\left(\bar{a}+\bar{b} x_{3}^{\prime}+\bar{c} x_{4}^{\prime}\right), \\
\left(d+e x_{3}+f x_{4}\right)-x_{3}\left(a+b x_{3}+c x_{4}\right) & \\
& =-\left(\bar{d}+\bar{e} x_{3}^{\prime}+\bar{f} x_{4}^{\prime}\right)+x_{3}^{\prime}\left(\bar{d}+\bar{b} x_{3}^{\prime}+\bar{c} x_{4}^{\prime}\right) .
\end{aligned}
$$

From (29) we get

$$
\begin{aligned}
x_{3}^{\prime} & =\left(d+e x_{3}+f x_{4}\right) /\left(a+b x_{3}+c x_{4}\right), \\
x_{3} & =\left(\bar{d}+\bar{e} x_{3}^{\prime}+\bar{f} x_{4}^{\prime}\right) /\left(\bar{a}+\bar{b} x_{3}^{\prime}+\bar{c} x_{4}^{\prime}\right),
\end{aligned}
$$


but these are precisely the equations $\S 3,(1),(2)$ by which the involutorial transformation $I_{n}$ was defined.

Hence we see that a $(1,2)$ correspondence of the type given by equations (26) leads in general to a special type of involutorial transformation of order $2 n-1$ with a $(2 n-3)$-fold line. If however conditions are imposed that $\bar{c} x_{3}-\bar{f}$ be a factor of the numerator of the expression for $x_{4}^{\prime}$, then $\bar{c} x_{3}-\bar{f}$ is a factor of $x_{1}^{\prime}, x_{2}^{\prime}, x_{3}^{\prime}$, and $x_{4}^{\prime}$ and we have an $I_{n}$ with an $(n-1)$-fold line. We have therefore proved the

THEOREM. An involutorial transformation in $S_{3}$ of order $n$ with an $(n-1)$ fold line is rational.

9. Image of a general line in $(y)$. A line $y_{3}=A y_{1}+B y_{2}, y_{4}=C y_{1}+D y_{2}$ in the $(y)$ space is transformed by the correspondence into the $C_{n+4}$ given parametrically by the equations

$$
\begin{aligned}
& X_{1}=x_{1}^{2} x_{2}\left(c V-f x_{1} x_{2}\right), \\
& X_{2}=x_{1} x_{2}^{2}\left(c V-f x_{1} x_{2}\right), \\
& X_{3}=-\bar{V}\left(c V-f x_{1} x_{2}\right), \\
& X_{4}=d x_{1}^{2} x_{2}^{2}-x_{1} x_{2}(a V+e \bar{V})+b V \bar{V},
\end{aligned}
$$

where

$$
\begin{aligned}
& V=x_{1}\left(C x_{1}^{2}+D x_{2}^{2}\right)+x_{2}\left(A x_{1}^{2}+B x_{2}^{2}\right), \\
& \bar{V}=x_{1}\left(C x_{1}^{2}+D x_{2}^{2}\right)-x_{2}\left(A x_{1}^{2}+B x_{2}^{2}\right) .
\end{aligned}
$$

In the case of the $I_{3}$ the curve is a $C_{7}$ of the form

$$
\begin{aligned}
& X_{1}=b_{0} x_{1}^{8} x_{2} V, \\
& X_{2}=b_{0} x_{1}^{2} x_{2}^{2} V, \\
& X_{3}=-b_{0} x_{1} V \bar{V}, \\
& X_{4}=x_{2}\left[a_{1} V \bar{V}-x_{1}\left(\sigma_{1} \sigma_{2} V+\rho_{1} \rho_{2} \bar{V}\right)\right] .
\end{aligned}
$$

If we put $V=0$ in (32), there are three values of the parameter $x_{2} / x_{1}$ all giving the point $(0,0,0,1)$. If we put $\bar{V}=0$, we get three distinct points in the invariant plane $x_{3}=0$. When $x_{1}=0$, we again have the point $(0,0,0,1)$ and furthermore the $C_{7}$ is tangent to $l$ at that point with $x_{1}=0$ as the osculating plane. When we put $x_{2}=0$, we have the point $(0,0,1,0)$. Hence the $C_{7}$ has a fourfold point $(0,0,0,1)$ at which it is also tangent to $l$ and passes through the point $(0,0,1,0)$.

In the general case there are $n+1$ values of $x_{2} / x_{1}$ due to the vanishing of $\left(c V-f x_{1} x_{2}\right)$ which give the point $(0,0,0,1)$. When $x_{1}=0$ or $x_{2}=0$, we get 
two definite points on $l$ at which the $C_{n+4}$ is tangent to the planes $x_{1}=0, x_{2}=0$ respectively. A plane of the pencil $y_{2}=k^{2} y_{1}$ has for images the two planes $x_{2}= \pm k x_{1}$ which meet the $C_{n+4}$ in the two images of the point in which $y_{2}=k^{2} y_{1}$ meets the line of which the $C_{n+4}$ is the image.

10. Image of a line in $(y)$ which meets $l^{\prime}$. Any line in $(y)$ meeting $l^{\prime}$ may be defined by

$$
y_{2}=k^{2} y_{1}, \quad A y_{1}+B y_{2}+C y_{3}+D y_{4}=0 .
$$

The image in $(x)$ of such a line is a pair of conics each belonging to a net in the planes $x_{2}= \pm k x_{1}$. In the plane $x_{2}=k x_{1}$ the net has the form

$$
\begin{gathered}
2 x_{1}\left(a_{11} x_{1}+b_{11} x_{3}+c_{11} x_{4}\right)\left(A+k^{2} B\right)+x_{1}\left(d_{11} x_{1}+e_{11} x_{3}+f_{11} x_{4}\right)(C+k D) \\
+x_{3}\left(a_{11} x_{1}+b_{11} x_{3}+c_{11} x_{4}\right)(C-k D)=0,
\end{gathered}
$$

where $a_{11}=a(1, k)$, etc. The conics of the net pass through the fixed points

$$
\begin{aligned}
& x_{1}=x_{2}=b_{11} x_{3}+c_{11} x_{4}=0, \\
& x_{1}=x_{2}=x_{3}=0, \\
& x_{2}-k x_{1}=a_{11} x_{1}+b_{11} x_{3}+c_{11} x_{4}=d_{11} x_{1}+e_{11} x_{3}+f_{11} x_{4}=0 .
\end{aligned}
$$

Two lines in $y_{2}=k^{2} y_{1}$ have for images a pair of conics of each net; the point of intersection of the two lines corresponds to the two free intersections of the two pairs of conics.

I In the case of the invariant plane $y_{2}=0$, the lines in $y_{2}=0$ correspond to a pencil of conics in the plane $x_{2}=0$ given by

$2 A x_{1}\left(a_{10} x_{1}+b_{10} x_{3}+c_{10} x_{4}\right)+C\left[x_{1}\left(d_{10} x_{1}+e_{10} x_{3}+f_{10} x_{4}\right)+x_{3}\left(a_{10} x_{1}+b_{10} x_{3}+c_{10} x_{4}\right)\right]=0$ where $a_{10}=a(1,0)$, etc. The pencil of conics has the three fixed points

$$
\begin{aligned}
& x_{1}=x_{2}=b_{10} x_{3}+c_{10} x_{4}=0, \\
& x_{1}=x_{2}=x_{3}=0, \\
& x_{2}=a_{10} x_{1}+b_{10} x_{3}+c_{10} x_{4}=d_{10} x_{1}+e_{10} x_{3}+f_{10} x_{4}=0 .
\end{aligned}
$$

The variable point of intersection of the net of conics is in this case replaced by the direction of the tangent to

$$
x_{1}\left(d_{10} x_{1}+e_{10} x_{3}+f_{10} x_{4}\right)+x_{3}\left(a_{10} x_{1}+b_{10} x_{3}+c_{10} x_{4}\right)=0
$$

at the point $x_{1}=x_{2}=b_{10} x_{3}+c_{10} x_{4}=0$. Hence this point is an invariant point the image of which in $(y)$ is $y_{2}=0$. Similarly the plane $y_{1}=0$ is the image of the invariant point $x_{1}=x_{2}=b_{01} x_{3}+c_{01} x_{4}=0$. The surface of branch points in the $(y)$ space consists of the two planes $y_{1}=0, y_{2}=0$, and the corresponding surface of coincidences in $(x)$ reduces to the two invariant points. 
ADDENDUM

In a recently published article* Snyder discusses involutorial birational transformations contained multiply in a linear line complex and suggests that they are probably irrational. The transformation he considers is of order $2 k$ with a $(2 k-1)$-fold line $x_{3}=x_{4}=0$, and $2 k-1$ fundamental points lying on the line $x_{1}=x_{2}=0$, and so is a special case of the involutorial transformations studied in this paper. The equations of the $I_{2 k}$ are given as

$$
\begin{aligned}
& x_{1}^{\prime}=\left(x_{3}^{2 k-1}+x_{4}^{2 k-1}\right) x_{1}, \\
& x_{2}^{\prime}=\left(x_{3}^{2 k-1}+x_{4}^{2 k-1}\right) x_{2}, \\
& x_{3}^{\prime}=\left(x_{4}^{2 k-1}-x_{3}^{2 k-1}\right) x_{3}, \\
& x_{4}^{\prime}=\left(x_{3}^{2 k-1}-x_{4}^{2 k-1}\right) x_{4} .
\end{aligned}
$$

This involutorial transformation may be mapped, as in the general case, on ordinary space by the $(1,2)$ correspondence given by the equations

$$
\begin{aligned}
& y_{1}=x_{1} x_{4} x_{3}{ }^{2 k-1}, \\
& y_{2}=x_{2} x_{4} x_{3}^{2 k-1}, \\
& y_{3}=x_{3}{ }^{2}\left(x_{3}{ }^{2-1}-x_{4}^{2 k-1}\right), \\
& y_{4}=x_{4}{ }^{2}\left(x_{3}{ }^{2-1}-x_{4}{ }^{2 k-1}\right),
\end{aligned}
$$

and is therefore rational.

* V. Snyder, The simplest involutorial transformation contained multiply in a line complex, Bulletin of the American Mathematical Society, vol. 36 (1930), pp. 89-93.

Cornell University,

Itraca, $\mathbf{N}$. Y. 